\title{
NUMERICAL SOLUTION OF NON-HOMOGENOUS FRACTIONAL OSCILLATOR EQUATION IN INTEGRAL FORM
}

\author{
MARIUSz Ciesielski \\ Czestochowa University of Technology, Institute of Computer and Information Sciences, Częstochowa, Poland \\ e-mail: mariusz.ciesielski@icis.pcz.pl \\ TOMAsz BŁAszCZYK \\ Czestochowa University of Technology, Institute of Mathematics, Częstochowa, Poland \\ e-mail: tomasz.blaszczyk@im.pcz.pl
}

In this paper, a non-homogenous fractional oscillator equation in finite time interval is considered. The fractional equation with derivatives of order $\alpha \in(0,1]$ is transformed into its corresponding integral form. Next, a numerical solution of the integral form of the considered equation is presented. In the final part of this paper, some examples of numerical solutions of the considered equation are shown.

Keywords: fractional oscillator equation, fractional integral equation, numerical solution

\section{Introduction}

Fractional differential equations have numerous applications in physics, engineering and biology. There is a number of monographs (Atanackovic et al., 2014; Baleanu et al., 2012; Hilfer, 2000; Kilbas et al., 2006; Klimek, 2009; Leszczynski, 2011; Magin, 2006; Malinowska and Torres, 2012; Podlubny, 1999) and a huge number of papers (Agrawal, 2002; Ciesielski and Leszczynski, 2006; Katsikadelis, 2012; Klimek, 2001; Riewe, 1996; Sumelka, 2014; Sumelka and Blaszczyk, 2014; Zhang et al., 2014) that cover various problems in fractional calculus. The list is large and is growing rapidly. An important issue is that the derivative of fractional order at any point of the domain has a local property only when the order is an integer number. For non-integer cases, the fractional derivative is a nonlocal operator and depends on the past values of the function (left derivative) or future ones (right derivative).

There are two different ways to formulate differential equations containing derivatives of fractional order. The first way is simply to replace integer order derivatives in differential equations by fractional ones. The second one relies on modifying the variational principle by replacing the integer order derivative by a fractional one. This leads to fractional differential equations which are known in the literature as the fractional Euler-Lagrange equations. It involves different types of Lagrangians, e.g., depending on Riemann-Liouville or Caputo fractional derivatives, fractional integrals, and mixed integer-fractional order operators (Agrawal et al., 2011; Almeida and Malinowska, 2012; Baleanu et al., 2014; Klimek et al., 2014; Odzijewicz et al., 2012). This second approach, in recent years, seems to become increasingly important.

The main feature of the fractional Euler-Lagrange equations is that these equations involve simultaneously the left and right derivatives of fractional order. This is also a fundamental problem in finding solutions to equations of a variational type (Baleanu et al., 2012; Klimek, 2009). Consequently, numerous studies have been devoted to numerical schemes for the fractional Euler-Lagrange equations (Blaszczyk et al., 2011; Blaszczyk and Ciesielski, 2014, 2015a,b; Bourdin et al., 2013; Pooseh et al., 2013; Xu and Agrawal, 2014). 
In this paper, we present a numerical solution of the non-homogenous fractional oscillator equation in a finite time interval.

\section{Preliminaries}

We consider a variational differential equation containing fractional derivatives of order $\alpha \in(0,1]$ in the finite time interval $t \in[0, b]$

$$
{ }^{C} D_{b^{-}}^{\alpha} D_{0^{+}}^{\alpha} x(t)-\omega^{2 \alpha} x(t)=f(t)
$$

where $\omega>0, x(t)$ is an unknown function and $f(t)$ is a given function. Equation (2.1) is supplemented by the following boundary conditions

$$
x(0)=0 \quad x(b)=L
$$

In this type of equation (derived from the Lagrangian with fractional derivative and the properties of the considered variational problem (Klimek, 2009) we assume $x(0)=0$ on the left boundary.

We recall some definitions and properties of the fractional operators (Kilbas et al., 2006; Oldham and Spanier, 1974; Podlubny, 1999). The left and right Riemann-Liouville fractional derivatives of order $\alpha \in(0,1)$ are defined by

$$
\begin{aligned}
D_{0^{+}}^{\alpha} x(t) & :=D I_{0^{+}}^{1-\alpha} x(t) \\
D_{b^{-}}^{\alpha} x(t) & :=-D I_{b^{-}}^{1-\alpha} x(t)
\end{aligned}
$$

and the left and right Caputo derivatives are defined as follows

$$
\begin{aligned}
{ }^{C} D_{0^{+}}^{\alpha} x(t) & :=I_{0^{+}}^{1-\alpha} D x(t) \\
{ }^{C} D_{b^{-}}^{\alpha} x(t) & :=-I_{b^{-}}^{1-\alpha} D x(t)
\end{aligned}
$$

where $D$ is an operator of the first order derivative and the operators $I_{0^{+}}^{\alpha}$ and $I_{b^{-}}^{\alpha}$ are respectively the left and right fractional integrals of order $\alpha>0$ defined by

$$
\begin{aligned}
& I_{0^{+}}^{\alpha} x(t):=\frac{1}{\Gamma(\alpha)} \int_{0}^{t} \frac{x(\tau)}{(t-\tau)^{1-\alpha}} d \tau \quad(t>0) \\
& I_{b^{-}}^{\alpha} x(t):=\frac{1}{\Gamma(\alpha)} \int_{t}^{b} \frac{x(\tau)}{(\tau-t)^{1-\alpha}} d \tau \quad(t<b)
\end{aligned}
$$

If $\alpha=1$ then $D_{0^{+}}^{1} x={ }^{C} D_{0^{+}}^{1} x=x^{\prime}$ and $D_{b^{-}}^{1} x={ }^{C} D_{b^{-}}^{1} x=-x^{\prime}$. The relations between the Caputo and Riemann-Liouville derivatives are of the form

$$
\begin{aligned}
& { }^{C} D_{0^{+}}^{\alpha} x(t)=D_{0^{+}}^{\alpha} x(t)-\frac{t^{-\alpha}}{\Gamma(1-\alpha)} x(0) \\
& { }^{C} D_{b^{-}}^{\alpha} x(t)=D_{b^{-}}^{\alpha} x(t)-\frac{(b-t)^{-\alpha}}{\Gamma(1-\alpha)} x(b)
\end{aligned}
$$

The composition rules of the fractional operators (for $\alpha \in(0,1])$ looks as follows

$$
\begin{aligned}
I_{0^{+}}^{\alpha}{ }^{C} D_{0^{+}}^{\alpha} x(t) & =x(t)-x(0) \\
I_{b^{-}}^{\alpha}{ }^{C} D_{b^{-}}^{\alpha} x(t) & =x(t)-x(b)
\end{aligned}
$$


and the fractional integral of a constant $C$

$$
I_{0^{+}}^{\alpha} C=C \frac{t^{\alpha}}{\Gamma(1+\alpha)}
$$

In particular, when $\alpha=1$, then ${ }^{C} D_{b^{-}}^{1} D_{0^{+}}^{1}=-D^{2}$ and Eq. (2.1) becomes

$$
-D^{2} f(t)-\omega^{2} x(t)=f(t)
$$

and its analytical solution with respect to boundary conditions (2.2) has the form

$$
x(t)=\frac{\sin (\omega t)}{\sin (\omega b)}[L+F(b)]-F(t)
$$

where

$$
F(t)=\frac{1}{\omega} \int_{0}^{t} f(\tau) \sin (\omega(t-\tau)) d \tau
$$

This solution we will apply to verifiction of numerical results obtained by our method for the case $\alpha=1$.

\section{Equivalent integral form}

The first step of our method is to transform a differential equation into an integral equation. We integrate Eq. (2.1) twice: the first time by using right fractional integral operator $(2.5)_{2}$ and the second time by using left fractional integral operator $(2.5)_{1}$. Finally, we obtain

$$
I_{0^{+}}^{\alpha} I_{b^{-}}^{\alpha}{ }^{C} D_{b^{-}}^{\alpha} D_{0^{+}}^{\alpha} x(t)-\omega^{2 \alpha} I_{0^{+}}^{\alpha} I_{b^{-}}^{\alpha} x(t)=I_{0^{+}}^{\alpha} I_{b^{-}}^{\alpha} f(t)
$$

Next, we use the composition rule of operators $(2.7)_{2}$ and get

$$
I_{0^{+}}^{\alpha}\left(D_{0^{+}}^{\alpha} x(t)-\left.D_{0^{+}}^{\alpha} x(t)\right|_{t=b}\right)-\omega^{2 \alpha} I_{0^{+}}^{\alpha} I_{b^{-}}^{\alpha} x(t)=I_{0^{+}}^{\alpha} I_{b^{-}}^{\alpha} f(t)
$$

In the above equation there occurs the value $\left.D_{0^{+}}^{\alpha} x(t)\right|_{t=b}$, and it is unknown at this stage. Here, we treat it as a constant. By using property $(2.7)_{1}$ and the assumption $x(0)=0$ in the boundary condition, and the fractional integral of constant (2.8) we obtain the following form of the considered equation

$$
x(t)-\left.D_{0^{+}}^{\alpha} x(t)\right|_{t=b} \frac{t^{\alpha}}{\Gamma(\alpha+1)}-\omega^{2 \alpha} I_{0^{+}}^{\alpha} I_{b^{-}}^{\alpha} x(t)=I_{0^{+}}^{\alpha} I_{b^{-}}^{\alpha} f(t)
$$

The unknown value $\left.D_{0^{+}}^{\alpha} x(t)\right|_{t=b}$ in the above equation can be determined on the basis of the boundary condition. Substituting the value $t=b$ into Eq. (3.3)

$$
x(b)-\left.D_{0^{+}}^{\alpha} x(t)\right|_{t=b} \frac{b^{\alpha}}{\Gamma(\alpha+1)}-\left.\omega^{2 \alpha} I_{0^{+}}^{\alpha} I_{b^{-}}^{\alpha} x(t)\right|_{t=b}=\left.I_{0^{+}}^{\alpha} I_{b^{-}}^{\alpha} f(t)\right|_{t=b}
$$

we obtain

$$
\left.D_{0^{+}}^{\alpha} x(t)\right|_{t=b}=\frac{\Gamma(\alpha+1)}{b^{\alpha}}\left[x(b)-\left.\omega^{2 \alpha} I_{0^{+}}^{\alpha} I_{b^{-}}^{\alpha} x(t)\right|_{t=b}-\left.I_{0^{+}}^{\alpha} I_{b^{-}}^{\alpha} f(t)\right|_{t=b}\right]
$$

Next, we put the right-hand side of formula (3.5) into Eq. (3.3) and get the integral form of Eq. (2.1)

$$
x(t)-\omega^{2 \alpha}\left[I_{0^{+}}^{\alpha} I_{b^{-}}^{\alpha} x(t)-\left.\left(\frac{t}{b}\right)^{\alpha} I_{0^{+}}^{\alpha} I_{b^{-}}^{\alpha} x(t)\right|_{t=b}\right]=\left(\frac{t}{b}\right)^{\alpha}\left[L-\left.I_{0^{+}}^{\alpha} I_{b^{-}}^{\alpha} f(t)\right|_{t=b}\right]+I_{0^{+}}^{\alpha} I_{b^{-}}^{\alpha} f(t)
$$




\section{Numerical solution}

In this Section, we present a numerical scheme for Eq. (3.6). We introduce a homogeneous grid of $n+1$ nodes (with the constant time step $\Delta t=b / n$ ): $0=t_{0}<t_{1}<\ldots<t_{i}<t_{i+1}<\ldots<t_{n}=b$, and $t_{i}=i \Delta t, i=0,1, \ldots, n$. For every grid node $t_{i}$, we write the following equation

$$
\begin{array}{r}
x\left(t_{i}\right)-\omega^{2 \alpha}\left[\left.I_{0^{+}}^{\alpha} I_{b^{-}}^{\alpha} x(t)\right|_{t=t_{i}}-\left.\left(\frac{t_{i}}{t_{n}}\right)^{\alpha} I_{0^{+}}^{\alpha} I_{b^{-}}^{\alpha} x(t)\right|_{t=t_{n}}\right] \\
=\left(\frac{t_{i}}{t_{n}}\right)^{\alpha}\left[L-\left.I_{0^{+}}^{\alpha} I_{b^{-}}^{\alpha} f(t)\right|_{t=t_{n}}\right]+\left.I_{0^{+}}^{\alpha} I_{b^{-}}^{\alpha} f(t)\right|_{t=t_{i}}
\end{array}
$$

In order to simplify notation, we denote the values of functions $x(t)$ and $f(t)$ at the node $t_{i}$ by $x_{i}=x\left(t_{i}\right)$ and $f_{i}=f\left(t_{i}\right)$, respectively. The numerical problem is to approximate the values of $\left.I_{0^{+}}^{\alpha} I_{b^{-}}^{\alpha} x(t)\right|_{t=t_{i}}$ and $\left.I_{0^{+}}^{\alpha} I_{b^{-}}^{\alpha} f(t)\right|_{t=t_{i}}$, for $i=0,1, \ldots, n$, properly. In our previous works (Blaszczyk and Ciesielski, 2015a,b) we have determined the discrete form of the composition of operators. On the basis of these results, we present the final discrete forms

$$
\begin{aligned}
\left.I_{0^{+}}^{\alpha} I_{b^{-}}^{\alpha} x(t)\right|_{t=t_{i}} & \approx \sum_{j=0}^{i} u_{i, j}^{(\alpha)} \sum_{k=j}^{n} v_{j, k}^{(\alpha)} x_{k} \\
\left.I_{0^{+}}^{\alpha} I_{b^{-}}^{\alpha} f(t)\right|_{t=t_{i}} & \approx \sum_{j=0}^{i} u_{i, j}^{(\alpha)} \sum_{k=j}^{n} v_{j, k}^{(\alpha)} f_{k}
\end{aligned}
$$

where the coefficients $u_{i, j}^{(\alpha)}$ and $v_{j, k}^{(\alpha)}$ are as follows

$$
\begin{gathered}
u_{i, j}^{(\alpha)}=\frac{(\Delta t)^{\alpha}}{\Gamma(\alpha+2)} \begin{cases}0 & \text { for } i=0 \text { and } j=0 \\
(i-1)^{\alpha+1}-i^{\alpha+1}+i^{\alpha}(\alpha+1) & \text { for } i>0 \text { and } j=0 \\
(i-j+1)^{\alpha+1}-2(i-j)^{\alpha+1}+(i-j-1)^{\alpha+1} & \text { for } i>0 \text { and } 0<j<i \\
1 & \text { for } i>0 \text { and } j=i\end{cases} \\
v_{j, k}^{(\alpha)}=\frac{(\Delta t)^{\alpha}}{\Gamma(\alpha+2)} \begin{cases}0 & \text { for } j=n \text { and } k=n \\
(n-j-1)^{\alpha+1}-(n-j)^{\alpha+1}+(n-j)^{\alpha}(\alpha+1) & \text { for } j<n \text { and } k=n \\
(k-j+1)^{\alpha+1}-2(k-j)^{\alpha+1}+(k-j-1)^{\alpha+1} & \text { for } j<n \text { and } j<k<n \\
1 & \text { for } j<n \text { and } k=j\end{cases}
\end{gathered}
$$

One can observe that in order to compute the values of operators (4.2) at every node $t_{i}$, we need to use the values of functions at all nodes of the domain.

Now we present the numerical scheme of integral equation (3.6). If we substitute (4.2) into (4.1), then the solution can be written as a system of $n+1$ linear equations

$$
\begin{aligned}
x_{i} & -\omega^{2 \alpha}\left[\sum_{j=0}^{i} u_{i, j}^{(\alpha)} \sum_{k=j}^{n} v_{j, k}^{(\alpha)} x_{k}-\left(\frac{i}{n}\right)^{\alpha} \sum_{j=0}^{n} u_{n, j}^{(\alpha)} \sum_{k=j}^{n} v_{j, k}^{(\alpha)} x_{k}\right] \\
& =\left(\frac{i}{n}\right)^{\alpha}\left[L-\sum_{j=0}^{n} u_{n, j}^{(\alpha)} \sum_{k=j}^{n} v_{j, k}^{(\alpha)} f_{k}\right]+\sum_{j=0}^{i} u_{i, j}^{(\alpha)} \sum_{k=j}^{n} v_{j, k}^{(\alpha)} f_{k} \quad i=0, \ldots, n
\end{aligned}
$$


The above system can also be written in the matrix form

$$
\mathbf{A} \cdot \mathbf{x}=\mathbf{b}
$$

with the coefficients of matrices $\mathbf{A}$ and $\mathbf{b}$

$$
\begin{aligned}
& A_{i, j}=\left\{\begin{array}{ll}
1 & \text { if } i=j \\
0 & \text { if } i \neq j
\end{array} \quad-\omega^{2 \alpha}\left[\sum_{k=0}^{\min (i, j)} u_{i, k}^{(\alpha)} v_{k, j}^{(\alpha)}-\left(\frac{i}{n}\right)^{\alpha} \sum_{k=0}^{j} u_{n, k}^{(\alpha)} v_{k, j}^{(\alpha)}\right]\right. \\
& i=0, \ldots, n \quad j=0, \ldots, n \\
& b_{i}=\left(\frac{i}{n}\right)^{\alpha}\left[L-\sum_{j=0}^{n} u_{n, j}^{(\alpha)} \sum_{k=j}^{n} v_{j, k}^{(\alpha)} f_{k}\right]+\sum_{j=0}^{i} u_{i, j}^{(\alpha)} \sum_{k=j}^{n} v_{j, k}^{(\alpha)} f_{k} \quad i=0, \ldots, n
\end{aligned}
$$

respectively, and $\mathbf{x}=\left[x_{0}, x_{1}, \ldots, x_{n}\right]^{\mathrm{T}}$.
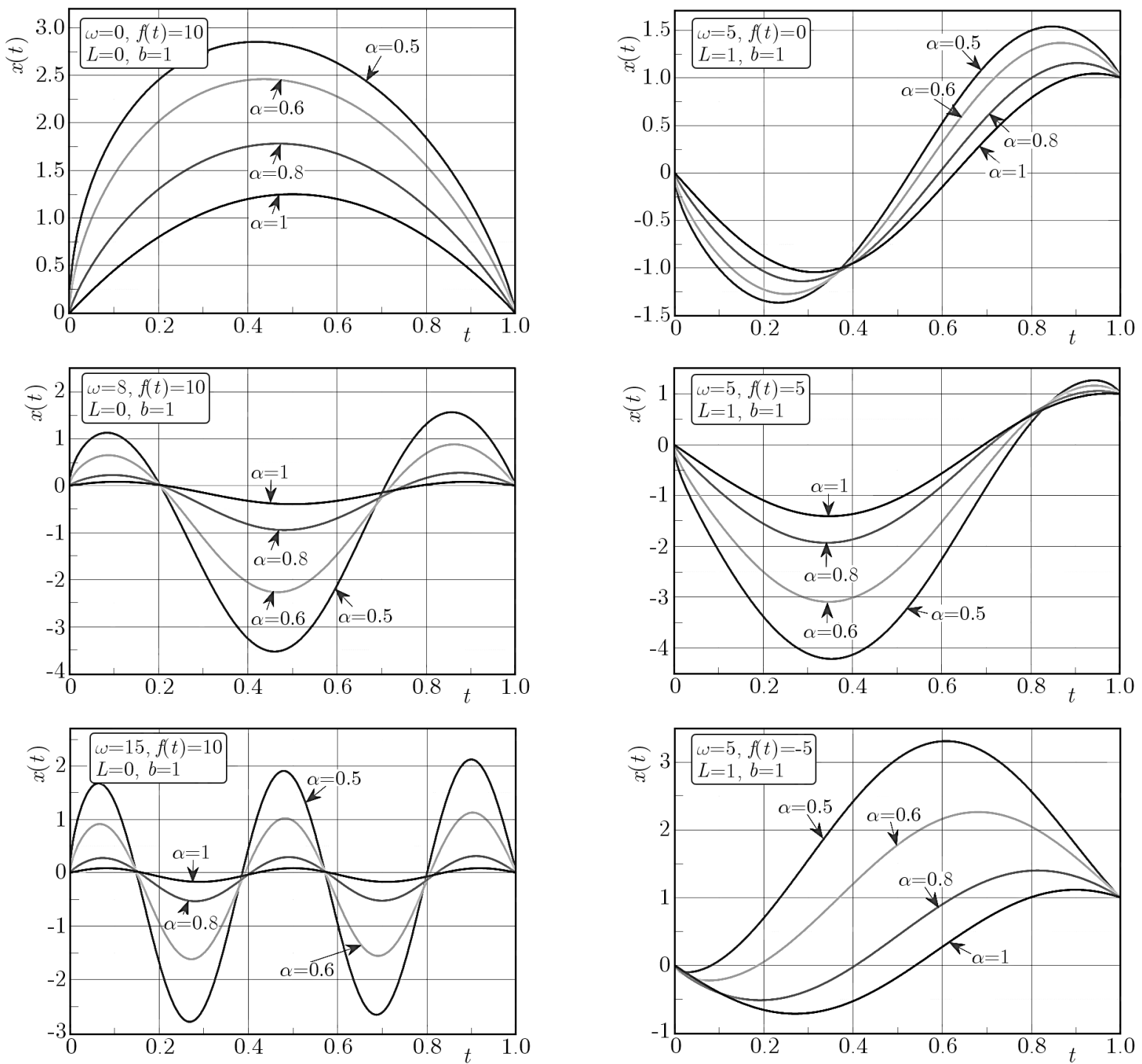

Fig. 1. Numerical solution of Eq. (2.1) for $\alpha \in\{0.5,0.6,0.8,1\}, b=1$, and different values of parameters $\omega, L$ and functions $f(t)$; left-side: different $\omega$ and $f(t)=10$; right-side: constant $\omega=5$ and different $f(t)$ 

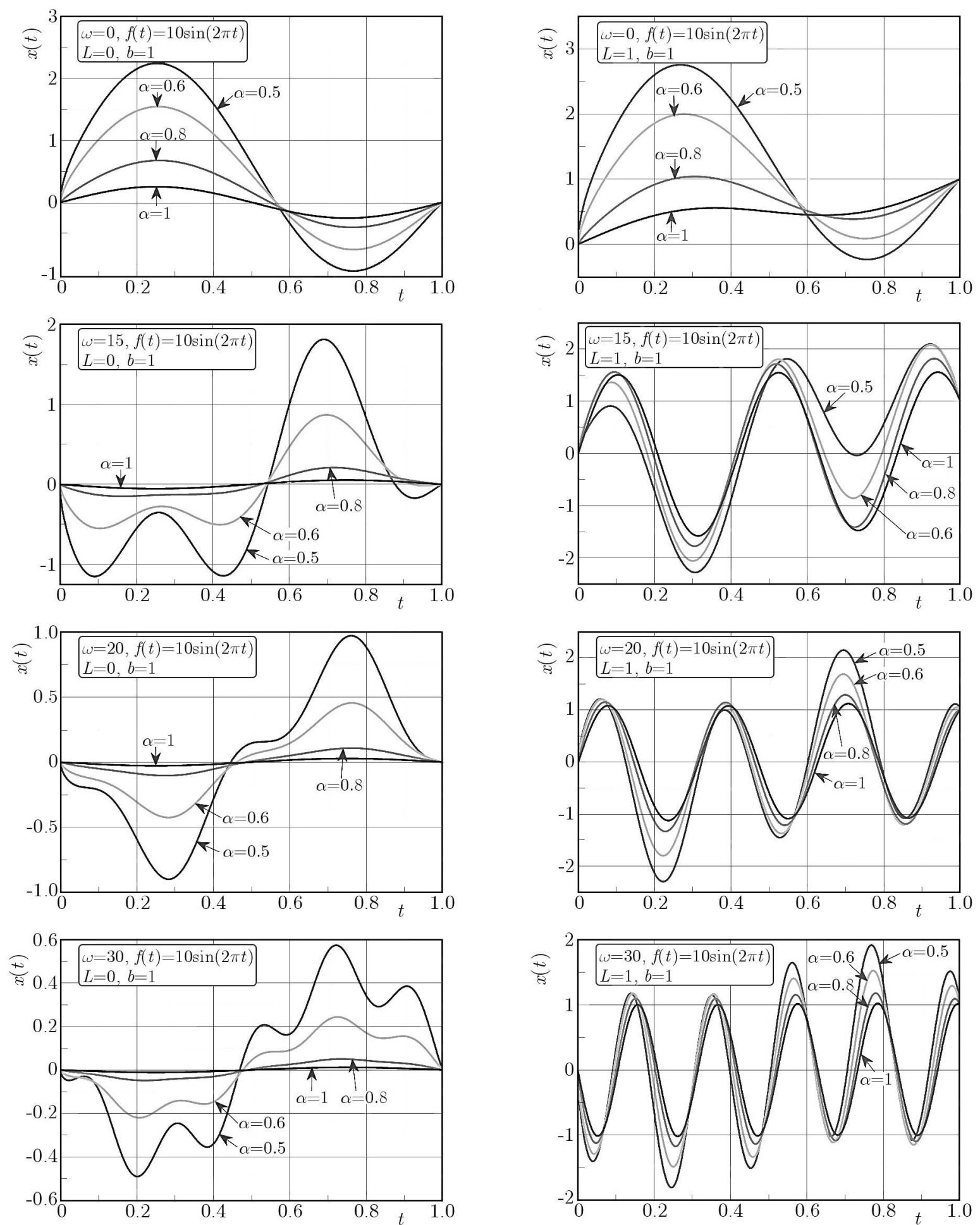

Fig. 2. Numerical solution of Eq. (2.1) for $\alpha \in\{0.5,0.6,0.8,1\}, f(t)=10 \sin (2 \pi t), b=1$, different values of parameter $\omega$, and $L=0$ (left-side), $L=1$ (right-side)

\section{Example of computations}

We present the results of computations obtained by our numerical scheme to the forced fractional oscillator equation. The system of linear equations (4.5) has been solved by the Gaussian elimination algorithm (the LUP decomposition (Press et al., 2007)). We present several exam- 
ples of calculations for different values of the parameters $\alpha, \omega$ and different functions $f$. In the examples we assume: $b=1$ and $L \in\{0,1\}$ in the right boundary condition. In Figs. 1 and 2 we present results of the numerical solution of Eq. (2.1). The values of the parameters used in the solution of equation (2.1) are given in plot legends. The time domain $t \in[0,1]$ has been divided into $n=1000$ subintervals in all examples.

\subsection{Numerical error analysis}

The discretization of differential or integral equations always entails certain errors. The knowledge of an analytical solution $x_{\text {anal }}(t)$ allows us to evaluate the errors of our numerical solution $x_{\text {num }}(t)$. We define the average error as

$$
E r r_{\text {avg }}=\frac{1}{m+1} \sum_{i=0}^{m}\left|x_{n u m}\left(t_{i}\right)-x_{\text {anal }}\left(t_{i}\right)\right| \quad \text { for } i=0, \ldots, m
$$

where $m$ is the number of measurement points.

Let us consider Eq. (2.1) with the parameters $\omega=0, L=0$ and the function $f(t)=(b-t)^{2-\alpha}$. In this case

$$
I_{0^{+}}^{\alpha} I_{b^{-}}^{\alpha} f(t)=I_{0^{+}}^{\alpha} I_{b^{-}}^{\alpha}(b-t)^{2-\alpha}=t^{\alpha} b^{2} \Gamma(3-\alpha) \sum_{j=0}^{2} \frac{(-1)^{j}}{\Gamma(3-j) \Gamma(\alpha+1+j)}\left(\frac{t}{b}\right)^{j}
$$

and the analytical solution (by using Eq. (3.6) and further simplifications) has form

$$
x_{\text {anal }}(t)=t^{\alpha} b^{2} \Gamma(3-\alpha) \sum_{j=0}^{2} \frac{(-1)^{j}\left[(t / b)^{j}-1\right]}{\Gamma(3-j) \Gamma(j+\alpha+1)}
$$

In Tables 1 and 2, we present the values of the analytical (exact) solution $x_{\text {anal }}(t)$ for $\alpha \in\{0.5,0.75\}$ at the selected values of nodes $t_{i}, i=0, \ldots, 10$ and the corresponding numerical values $x_{\text {num }}(t)$ calculated for different numbers of grid nodes $(n \in\{100,200,400,800\})$. The errors generated by numerical schema are also included in Tables 1 and 2. One can note that the error decreases with a decrease in the time step of the grid.

Table 1. Values of analytical and numerical solutions of Eq. (2.1) for $\alpha=0.5, \omega=0$, $f(t)=(b-t)^{2-\alpha}, b=1, L=0$

\begin{tabular}{|c|c|c|c|c|c|}
\hline$t_{i}$ & $x_{\text {anal }}\left(t_{i}\right)$ & $\begin{array}{c}x_{\text {num }}\left(t_{i}\right) \\
n=100\end{array}$ & $\begin{array}{c}x_{\text {num }}\left(t_{i}\right) \\
n=200\end{array}$ & $\begin{array}{c}x_{\text {num }}\left(t_{i}\right) \\
n=400\end{array}$ & $\begin{array}{c}x_{\text {num }}\left(t_{i}\right) \\
n=800\end{array}$ \\
\hline \hline 0 & 0.000000000000 & 0.000000000000 & 0.000000000000 & 0.000000000000 & 0.000000000000 \\
\hline 0.1 & 0.159378794072 & 0.159379032826 & 0.159378842522 & 0.159378803745 & 0.159378795979 \\
\hline 0.2 & 0.186040855728 & 0.186041292916 & 0.186040941860 & 0.186040872522 & 0.186040858974 \\
\hline 0.3 & 0.184034779322 & 0.184035363697 & 0.184034893461 & 0.184034801419 & 0.184034783568 \\
\hline 0.4 & 0.166968260457 & 0.166968963299 & 0.166968397209 & 0.166968286848 & 0.166968265515 \\
\hline 0.5 & 0.141421356237 & 0.141422156701 & 0.141421511713 & 0.141421386199 & 0.141421361972 \\
\hline 0.6 & 0.111541920371 & 0.111542800274 & 0.111542091205 & 0.111541953283 & 0.111541926669 \\
\hline 0.7 & 0.080319362547 & 0.080320302735 & 0.080319545231 & 0.080319397768 & 0.080319369292 \\
\hline 0.8 & 0.050087922696 & 0.050088897461 & 0.050088112561 & 0.050087959382 & 0.050087929735 \\
\hline 0.9 & 0.022768399153 & 0.022769357797 & 0.022768587015 & 0.022768435655 & 0.022768406192 \\
\hline 1 & 0.000000000000 & 0.000000000000 & 0.000000000000 & 0.000000000000 & 0.000000000000 \\
\hline \hline Err $_{\text {avg }}$ & - & $5.92466 \cdot 10^{-7}$ & $1.15654 \cdot 10^{-7}$ & $2.2385 \cdot 10^{-8}$ & $4.30118 \cdot 10^{-9}$ \\
\hline
\end{tabular}


Table 2. Values of analytical and numerical solutions of Eq. (2.1) for $\alpha=0.75, \omega=0$, $f(t)=(b-t)^{2-\alpha}, b=1, L=0$

\begin{tabular}{|c|c|c|c|c|c|}
\hline$t_{i}$ & $x_{\text {anal }}\left(t_{i}\right)$ & $\begin{array}{c}x_{\text {num }}\left(t_{i}\right) \\
n=100\end{array}$ & $\begin{array}{c}x_{\text {num }}\left(t_{i}\right) \\
n=200\end{array}$ & $\begin{array}{c}x_{\text {num }}\left(t_{i}\right) \\
n=400\end{array}$ & $\begin{array}{c}x_{\text {num }}\left(t_{i}\right) \\
n=800\end{array}$ \\
\hline \hline 0 & 0.000000000000 & 0.000000000000 & 0.000000000000 & 0.000000000000 & 0.000000000000 \\
\hline 0.1 & 0.067645942013 & 0.067646050586 & 0.067645965523 & 0.067645947053 & 0.067645943086 \\
\hline 0.2 & 0.094996909603 & 0.094997100277 & 0.094996949929 & 0.094996918109 & 0.094996911394 \\
\hline 0.3 & 0.105395550994 & 0.105395806812 & 0.105395604628 & 0.105395562237 & 0.105395553351 \\
\hline 0.4 & 0.104362700328 & 0.104363008356 & 0.104362764589 & 0.104362713751 & 0.104362703135 \\
\hline 0.5 & 0.095196902130 & 0.095197250210 & 0.095196974490 & 0.095196917206 & 0.095196905277 \\
\hline 0.6 & 0.080331627323 & 0.080332002341 & 0.080331705051 & 0.080331643482 & 0.080331630691 \\
\hline 0.7 & 0.061751765560 & 0.061752151496 & 0.061751845314 & 0.061751782104 & 0.061751769002 \\
\hline 0.8 & 0.041170523241 & 0.041170897406 & 0.041170600282 & 0.041170539178 & 0.041170526550 \\
\hline 0.9 & 0.020119449943 & 0.020119771392 & 0.020119515708 & 0.020119463484 & 0.020119452744 \\
\hline 1 & 0.000000000000 & 0.000000000000 & 0.000000000000 & 0.000000000000 & 0.000000000000 \\
\hline \hline Err $_{\text {avg }}$ & - & $2.42522 \cdot 10^{-7}$ & $5.03981 \cdot 10^{-8}$ & $1.04972 \cdot 10^{-8}$ & $2.19045 \cdot 10^{-9}$ \\
\hline
\end{tabular}

\section{Conclusions}

In this work, the method of solving of the non-homogenous fractional oscillator equation with derivatives of order $\alpha \in(0,1]$ has been presented. First, the equation has been transformed into integral form. Next, the numerical scheme for the integral form of the equation has been proposed. Analysing the solutions of the equation for different values of parameters $\alpha, \omega$, various boundary conditions and different functions $f(t)$ one can understand the influence of these parameters on the solution. One can note that if the value of $\alpha$ decreases then the amplitude of oscillations increases, and if the value of $\omega$ increases then the oscillation frequency increases as well. The analytical solution of this type of fractional differential equation (except for the case $\alpha=1$ ) is not yet known. Our proposed numerical method of solutions for $\alpha \rightarrow 1$ is consistent with the analytical solution for $\alpha=1$. This type of considered equation can be used in mathematical modelling of the oscillatory behavior of systems where the use of the classical oscillator equation is insufficient.

\section{References}

1. Agrawal O.P., 2002, Formulation of Euler-Lagrange equations for fractional variational problems, Journal of Mathematical Analysis and Applications, 272, 368-379

2. Agrawal O.P., Muslih S.I., Baleanu D., 2011, Generalized variational calculus in terms of multi-parameters fractional derivatives, Communications in Nonlinear Science and Numerical Simulation, 16, 4756-4767

3. Almeida R., Malinowska A.B., 2012, Generalized transversality conditions in fractional calculus of variations, Communications in Nonlinear Science and Numerical Simulation, 18, 3, 443-452

4. Atanackovic T.M., Pilipovic S., Stankovic B., Zorica D., 2014, Fractional Calculus with Applications in Mechanics: Vibrations and Diffusion Processes, ISTE-Wiley, London

5. Baleanu D., Trujillo J.J., 2008, On exact solutions of a class of fractional Euler-Lagrange equations, Nonlinear Dynamics, 52, 331-335

6. Baleanu D., Asad J.H., Petras I., 2014, Fractional Bateman-Feshbach Tikochinsky oscillator, Communications in Theoretical Physics, 61, 221-225

7. Baleanu D., Diethelm K., Scalas E., Trujillo J.J., 2012, Fractional Calculus Models and Numerical Methods, World Scientific, Singapore 
8. Blaszczyk T., Ciesielski M., 2014, Numerical solution of fractional Sturm-Liouville equation in integral form, Fractional Calculus and Applied Analysis, 17, 307-320

9. Blaszczyk T., Ciesielski M., 2015a, Fractional oscillator equation: analytical solution and algorithm for its approximate computation, Journal of Vibration and Control (in print), http://dx.doi.org/10.1177/1077546314566836

10. BlaszczyK T., Ciesielski M., 2015b, Fractional oscillator equation - transformation into integral equation and numerical solution, Applied Mathematics and Computation, 257, 428-435

11. Blaszczyk T., Ciesielski M., Klimek M., Leszczynski J., 2011, Numerical solution of fractional oscillator equation, Applied Mathematics and Computation, 218, 2480-2488

12. Bourdin L., Cresson J., Greff I., Inizan P., 2013, Variational integrator for fractional EulerLagrange equations, Applied Numerical Mathematics, 71, 14-23

13. Ciesielski M., Leszczynski J., 2006, Numerical solutions to boundary value problem for anomalous diffusion equation with Riesz-Feller fractional operator, Journal of Theoretical and Applied Mechanics, 44, 2, 393-403

14. Hilfer R., 2000, Applications of Fractional Calculus in Physics, World Scientific, Singapore

15. Katsikadelis J.T., 2012, Nonlinear dynamic analysis of viscoelastic membranes described with fractional differential models, Journal of Theoretical and Applied Mechanics, 50, 3, 743-753

16. Kilbas A.A., Srivastava H.M., Trujillo J.J., 2006, Theory and Applications of Fractional Differential Equations, Elsevier, Amsterdam

17. KLimeK M., 2001, Fractional sequential mechanics - models with symmetric fractional derivative, Czechoslovak Journal of Physics, 51, 1348-1354

18. KlimeK M., 2009, On Solutions of Linear Fractional Differential Equations of a Variational Type, Publishing Office of Czestochowa University of Technology, Czestochowa

19. Klimek M., Odzijewicz T., Malinowska A.B., 2014, Variational methods for the fractional Sturm-Liouville problem, Journal of Mathematical Analysis and Applications., 416, 402-426

20. Leszczynski J.S., 2011, An Introduction to Fractional Mechanics, Publishing Office of Czestochowa University of Technology, Czestochowa

21. Magin R.L., 2006, Fractional Calculus in Bioengineering, Begell House Inc, Redding

22. Malinowska A.B., Torres D.F.M., 2012, Introduction to the Fractional Calculus of Variations, Imperial College Press, London

23. Odzijewicz T., Malinowska A.B., Torres D.F.M., 2012, Fractional variational calculus with classical and combined Caputo derivatives, Nonlinear Analysis, Theory, Methods and Applications, 75, 1507-1515

24. Oldham K.B., Spanier J., 1974, The Fractional Calculus: Theory and Applications of Differentiation and Integration to Arbitrary Order, Academic Press, San Diego

25. Podlubny I., 1999, Fractional Differential Equations, Academic Press, San Diego

26. Pooseh S., Almeida R., Torres D.F.M., 2013, Discrete direct methods in the fractional calculus of variations, Computational and Applied Mathematics, 66, 668-676

27. Press W.H., Teukolsky S.A., Vetterling W.T., Flannery B.P., 2007, Numerical Recipes: The Art of Scientific Computing (3rd ed.), Cambridge University Press, New York

28. Riewe F., 1996, Nonconservative Lagrangian and Hamiltonian mechanics, Physical Review E, 53, 1890-1899

29. Sumelka W., 2014, A note on non-associated Drucker-Prager plastic flow in terms of fractional calculus, Journal of Theoretical and Applied Mechanics, 52, 2, 571-574

30. Sumelka W., Blaszczyk T., 2014, Fractional continua for linear elasticity, Archives of Mechanics, 66, 3, 147-172 
31. Xu Y., Agrawal O.P., 2014, Models and numerical solutions of generalized oscillator equations, Journal of Vibration and Acoustics, 136, 051005, 7pp

32. Zhang Y., Chen L., Reeves D.M., Sun H.G., 2014, A fractional-order temperedstable continuity model to capture surface water runoff, Journal of Vibration and Control, http://dx.doi.org//10.1177/1077546314557554

Manuscript received January 29, 2015; accepted for print May 20, 2015 